\title{
Estabelecimento de Parâmetros Reológicos na Obtenção de Compósitos Carbono/Fenólica.
}

\author{
Edson C. Botelho, Natália Scherbakoff, Mirabel C. Rezende
}

Resumo: As resinas fenólicas têm sido utilizadas no processamento de compósitos carbono/carbono, produzindo um material intermediário conhecido como compósito carbono/fenólica. O presente trabalho mostra os resultados obtidos de análises reológicas, cromatográficas e por calorimetria exploratória diferencial (DSC) de duas amostras de resinas fenólicas tipo novolaca e uma resol, visando escolher a matéria-prima e os parâmetros mais adequados ao processamento de compósitos carbono/fenólica. Os resultados obtidos levaram à escolha da resina resol para a impregnação de pré-formas de fibras de carbono e também fixaram a temperatura de impregnação do reforço de carbono com a resina para a obtenção do compósito intermediário.

Palavras-chave: Reologia, compósitos carbono/carbono, materiais carbonosos, compósitos carbono/fenólica.

\section{Introdução}

Os compósitos Carbono/Carbono $(\mathrm{C} / \mathrm{C})$ constituem uma classe de materiais que tem como matriz um material carbonoso e como elemento de reforço fibras de carbono ${ }^{[1-5]}$.

Atualmente, no setor espacial, o compósito $\mathrm{C} / \mathrm{C}$ está sendo utilizado como blindagem térmica em veículos de reentrada na atmosfera terrestre, assim como em gargantas de tubeiras de motor-foguete à base de propelente sólido ${ }^{[6-8]}$. No campo aeronáutico estes compósitos estão sendo usados em bordas de ataque de aeronaves supersônicas, em componentes estruturais que chegam a atingir temperaturas de até $1500^{\circ} \mathrm{C}$ e em sistemas de freios de aeronaves supersônicas militares e civis ${ }^{[6,7,9]}$. O potencial de frenagem desses sistemas é cerca de quatro a cinco vezes superior ao dos sistemas metálicos convencionais aumen- tando, assim, o seu desempenho ao ser acionado e por conseqüência a segurança da aeronave ${ }^{[9-11]}$. Este sistema apresenta, também, a vantagem de ser mais leve que os convencionais, chegando a aproximadamente 680 quilogramas, como no caso do Concorde, representando uma redução de peso de aproximadamente duas vezes. Em contrapartida, seu custo é mais elevado quando comparado ao dos sistemas de frenagem metálicos ${ }^{[1-3]}$.

$\mathrm{O}$ uso do compósito $\mathrm{C} / \mathrm{C}$ em aplicações espaciais e aeronáuticas se deve à baixa taxa de desgaste por ablação a temperaturas entre 1500 e $3000^{\circ} \mathrm{C}$ e aos seus adequados coeficientes de atrito e de calor específico nestas condições de trabalho ${ }^{[1-3]}$.

Um dos processos de obtenção dos $\mathrm{C} / \mathrm{C}$ tem como fase intermediária a obtenção de compósitos carbono/fenólica. Estes compósitos poliméricos reforçados com fibras de carbono, além de serem materiais 
precursores dos $\mathrm{C} / \mathrm{C}$, têm encontrado aplicações termoestruturais como suporte das gargantas de tubeiras de foguetes em $\mathrm{C} / \mathrm{C}$, na região de saída dos gases de queima do propelente e como proteção térmica nas regiões anterior e posterior à tubeira do motor-foguete ${ }^{[1,3,7]}$.

Estudos das condições de processamento das matérias-primas e de otimização do ferramental para a obtenção de artefatos utilizados como proteção térmica de foguetes, em compósito carbono/fenólica, se fazem necessários, devido às dimensões em que estas peças são obtidas e às exigências em serviço deste material ${ }^{[1,3]}$.

Sendo assim, o conhecimento prévio das características térmica, reológica e cromatográfica de prováveis precursores poliméricos fornece parâmetros para uma seleção mais apropriada do impregnante e do processo a serem utilizados no processamento dos compósitos carbono/fenólica e, por conseqüência, dos $\mathrm{C} / \mathrm{C}^{[12-19]}$.

\section{Precursores dos Compósitos C/C}

Os compósitos $\mathrm{C} / \mathrm{C}$ podem ser obtidos a partir de precursores ricos em átomos de carbono como piches, gases orgânicos e resinas termorrígidas e de fibras de carbono como reforço. Dentre os diversos tipos de resinas termorrígidas, as mais utilizadas para a produção dos materiais carbonosos avançados são as fenólicas e a furfurílica, devido aos seus teores de carbono fixo estarem em torno de $50 \%$ e do elevado grau de ligações cruzadas que estes polímeros apresentam $^{[20-27]}$.

As resinas fenólicas constituem um importante grupo de polímeros termorrígidos, formado de cadeias com grupamentos fenólicos intercalados por radicais metilênicos ${ }^{[21,28]}$. Estas resinas são formadas pela reação de condensação entre o fenol e o formaldeído, com a eliminação de água, podendo ocorrer ligações cruzadas de grupamentos metilênicos entre as cadeias, caso exista um excesso de formaldeído, também acompanhadas por eliminação de água ${ }^{[20-27]}$.

\section{Experimental}

Neste trabalho foram utilizados três tipos de resinas fenólicas líquidas de procedência nacional. A do tipo resol foi codificada como A e as duas do tipo novolaca com os códigos $\mathrm{B}$ e C. As resinas novolacas foram fornecidas com $9,5 \%(\mathrm{p} / \mathrm{p})$ do agente de cura hexametilenotetramina.

As resinas foram caracterizadas quanto aos teores de carbono fixo (baseado na norma ASTM D $3172-84^{[29]}$ ), voláteis (ASTM D 3175-73 ${ }^{[30]}$ ), cinzas (ASTM D 3174-82[31]), umidade via método Karl Fischer e medidas da viscosidade inicial das resinas utilizando-se um viscosímetro Brookfield ${ }^{[7]}$.

Os ensaios reológicos foram realizados em triplicata em um reômetro de tensão controlada da Reometric Scientific, série SR 200, com pratos paralelos. Para estas amostras foram obtidas isotermas a 100 e $110^{\circ} \mathrm{C}$, a uma freqüência constante de $1 \mathrm{rad} / \mathrm{s} \mathrm{e}$ tensão de 5,0 Pa. Para viabilizar as análises reológicas destas resinas foi necessário a redução dos seus teores de umidade para valores próximos a 3\%. Esta eliminação foi realizada com a utilização de um sistema de vácuo.

As análises por cromatografia de exclusão de tamanho (SEC - Size Exclusion Cromatography) foram realizadas em triplicata utilizando-se um sistema de colunas de 100, 100 e $500 \AA$, tetrahidrofurano (THF) como solvente e padrões de poliestireno (1.050-19.500 g/mol) e etilbenzeno (106 g/mol).

As análises térmicas por calorimetria exploratória diferencial das amostras foram realizadas em triplicata a uma taxa de aquecimento de $10^{\circ} \mathrm{C} / \mathrm{min}$ sob fluxo constante de nitrogênio em um equipamento da Perkin Elmer, modelo 7 Series Thermal Analysis System.

\section{Resultados e Discussão}

A Tabela 1 apresenta os resultados das determinações de carbono fixo, voláteis, cinzas, umidade e viscosidade dinâmica obtidos para as amostras de resinas fenólicas em estudo. O conhecimento destas características fornece os primeiros subsídios quanto aos parâmetros de processamento a serem utilizados e permite estimar algumas propriedades finais dos materiais a serem obtidos.

Quanto à viscosidade inicial, as amostras de resinas fenólicas resol A e novolaca B apresentaram valores próximos como indicado na Tabela 1, enquanto que a amostra de resina fenólica $\mathrm{C}$ apresentou um valor mais elevado, $1,00 \pm 0,01 \mathrm{~Pa}$.S.

Sabendo-se previamente que, no processamento 
Tabela 1. Valores de viscosidade, carbono fixo, umidade, cinzas e de materiais voláteis das resinas estudadas.

\begin{tabular}{lccccc}
\hline \multicolumn{1}{c}{ Resina } & Viscosidade (Pa.S) & Carbono fixo (\%) & Umidade $(\boldsymbol{\%})$ & Cinzas (\%) & Voláteis (\%) \\
\hline fenólica resol A & $0,74 \pm 0,01$ & $41,0 \pm 0,01$ & $7,50 \pm 0,01$ & $0,20 \pm 0,01$ & $51,3 \pm 0,01$ \\
fenólica novolaca B & $0,72 \pm 0,01$ & $28,8 \pm 0,01$ & $6,80 \pm 0,01$ & $0,19 \pm 0,01$ & $64,2 \pm 0,01$ \\
fenólica novolaca C & $1,00 \pm 0,01$ & $34,7 \pm 0,01$ & $6,80 \pm 0,01$ & $1,11 \pm 0,01$ & $57,4 \pm 0,01$ \\
\hline
\end{tabular}

de compósitos carbono/fenólica os valores de viscosidade inicial do polímero impregnante estão relacionados com a viabilidade de se obter uma boa impregnação do reforço, tem-se que menores valores de viscosidade favorecem esta etapa do processo.

Analisando-se, inicialmente, somente os valores de viscosidade dinâmica obtidos observa-se que todas as amostras mostraram-se adequadas para serem utilizadas como impregnante do reforço de carbono, pois estes valores são baixos e relativamente próximos.

Os teores de umidade das resinas fenólicas, como mostrados na Tabela 1, apresentaram-se inadequados ao processamento dos compósitos carbono/ fenólica e $\mathrm{C} / \mathrm{C}$ por favorecerem o aparecimento de vazios no material final, além de inviabilizarem as análises reológicas. Em função destes teores, foi necessário reduzir esta quantidade de água para no máximo $3,0 \%$, de modo que os voláteis gerados durante as análises reológicas não prejudicassem os resultados.

Os valores de carbono fixo das amostras das resinas novolacas $\mathrm{B}$ e $\mathrm{C}$, quando comparados aos da resina resol A, apresentaram-se menores, com o conseqüente aumento das porcentagens de voláteis. Estes resultados indicam que a resina resol A é a mais adequada no processamento de compósitos carbono/ fenólica, quando estes são precursores de compósitos $\mathrm{C} / \mathrm{C}$, pois o seu rendimento em carbono após o tratamento térmico de carbonização, ou seja para temperaturas superiores a $1000^{\circ} \mathrm{C}$, na ausência de oxigênio, aumentará a eficiência do processo de densificação das pré-formas de fibras de carbono.

A análise dos teores de cinzas mostra que a resina $\mathrm{C}$ apresentou o maior valor de resíduo inorgânico. Esta observação se faz importante quando o processamento de compósitos carbono/carbono visa estudos de compatibilidade biológica intracorpóreos, onde a presença de metais citotóxicos pode iniciar reações alérgicas com os tecidos vivos, mascarando os testes de biocompatibilidade do material e/ou do artefato médico em estudo.
Correlacionando os valores de carbono fixo, viscosidade dinâmica e cinzas tem-se que a resina resol A apresenta as melhores características para ser utilizada como precursora dos compósitos carbono/ fenólica e $\mathrm{C} / \mathrm{C}$.

A Figura 1 é representativa dos cromatogramas de exclusão por tamanho obtidos para todas as amostras analisadas. Como pode ser observado nesta figura, os
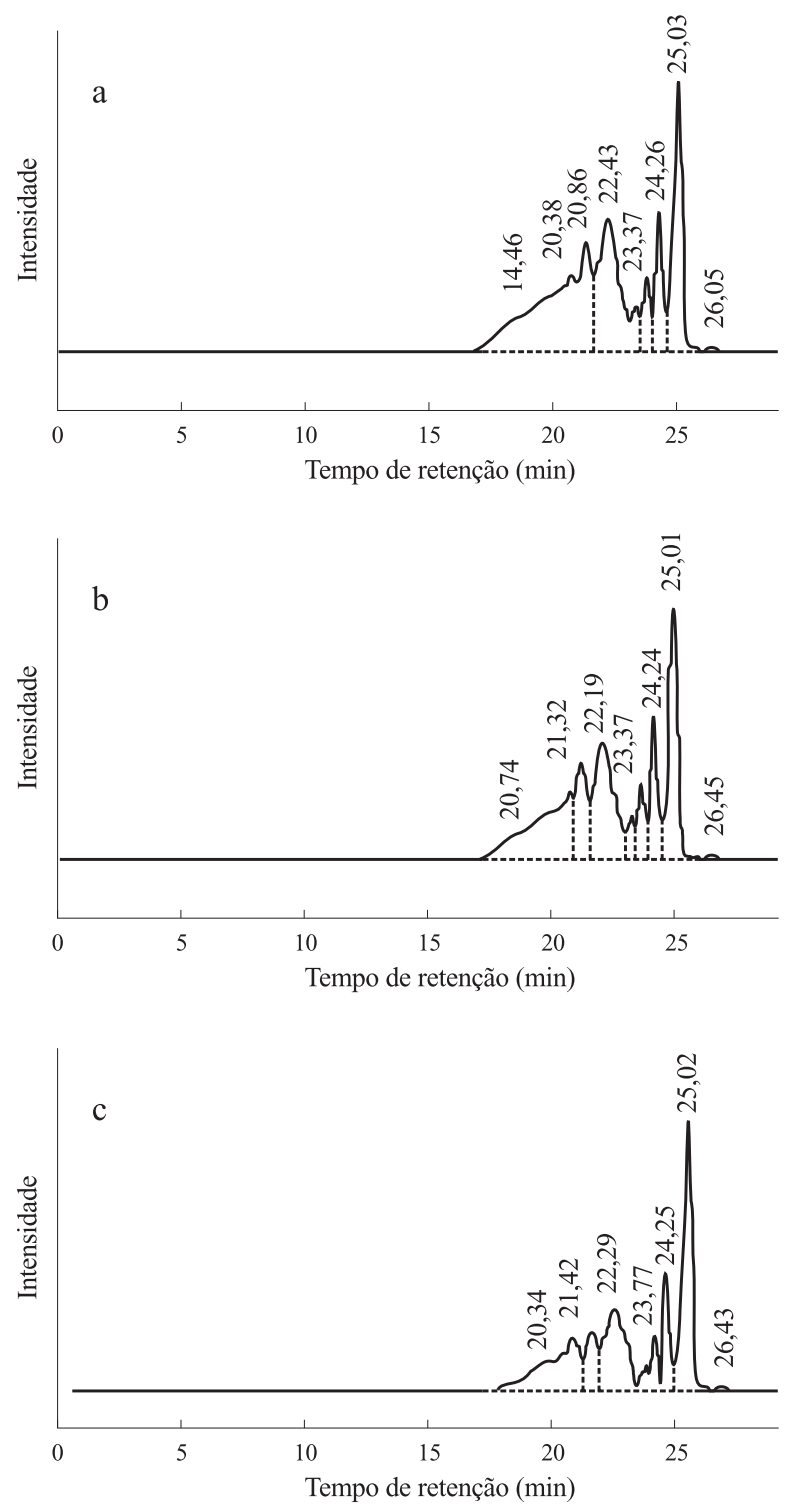

Figura 1. Cromatograma de exclusão por tamanho para as resinas resol A (a), novolaca B (b) e novolaca C (c). 


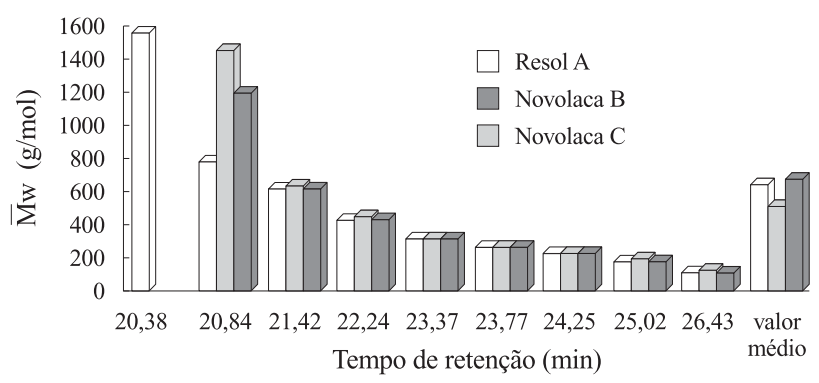

Figura 2. Histograma da distribuição do peso molecular médio versus tempo de retenção das resinas.

cromatogramas apresentaram um perfil semelhante quanto à distribuição de tamanho molecular, diferindo ligeiramente para a amostra de resina resol que mostrou um alargamento da banda abaixo do tempo de 20,9 min. Este comportamento pode ser melhor observado na Figura 2, onde o histograma compara a distribuição de tamanho molecular versus o tempo de retenção das amostras de resina, mostrando a presença de uma fração de maior tamanho molecular no tempo de aproximadamente 20,4 min para a resina resol A.

A Figura 3 mostra as curvas de DSC representativas das amostras de resina resol A e novolacas B e $\mathrm{C}$ e a Tabela 2 apresenta os valores das temperaturas de início, final e da metade da reação de polimerização $\left(\mathrm{T}_{50 \%}\right)$ obtidos desta figura.

Observa-se na Tabela 2 que as resinas fenólicas analisadas apresentaram as temperaturas de início e de final de reação próximas, porém a temperatura indica a metade da polimerização $(50 \%)$ variou devido às velocidades de reação de cura diferenciadas das resinas fenólicas $\mathrm{A}, \mathrm{B}$ e $\mathrm{C}$.

A correlação dos valores de temperatura de $50 \%$ de conversão com os de carbono fixo e as análises cromatográficas permite concluir que para as resinas em estudo, o maior valor de carbono fixo não se deve à presença significativa de frações de maior peso molecular, mas a uma cinética de polimerização diferenciada, onde menores valores de $\mathrm{T}_{50 \%}$ favorecem um maior ganho em carbono após a carbonização.

Com base nos resultados de DSC tem-se que a faixa de temperatura de início de polimerização das resinas em estudo encontra-se entre 106 e $110^{\circ} \mathrm{C}$. Em função destes valores as temperaturas escolhidas para a obten-

Tabela 2. Temperaturas de início, metade e final de polimerização para as amostras estudadas.

\begin{tabular}{cccc}
\hline Resina & $\mathbf{T}_{\text {iń́io }}\left({ }^{\mathbf{O}} \mathbf{C}\right)$ & $\mathbf{T}_{\mathbf{5 0 \%}}\left({ }^{\mathbf{}} \mathbf{C}\right)$ & $\mathbf{T}_{\text {final }}\left({ }^{\circ} \mathbf{C}\right)$ \\
\hline Resol A & $110 \pm 1,00$ & $128 \pm 1,00$ & $175 \pm 1,00$ \\
Novolaca B & $109 \pm 1,00$ & $151 \pm 1,00$ & $176 \pm 1,00$ \\
Novolaca C & $106 \pm 1,00$ & $131 \pm 1,00$ & $172 \pm 1,00$ \\
\hline
\end{tabular}
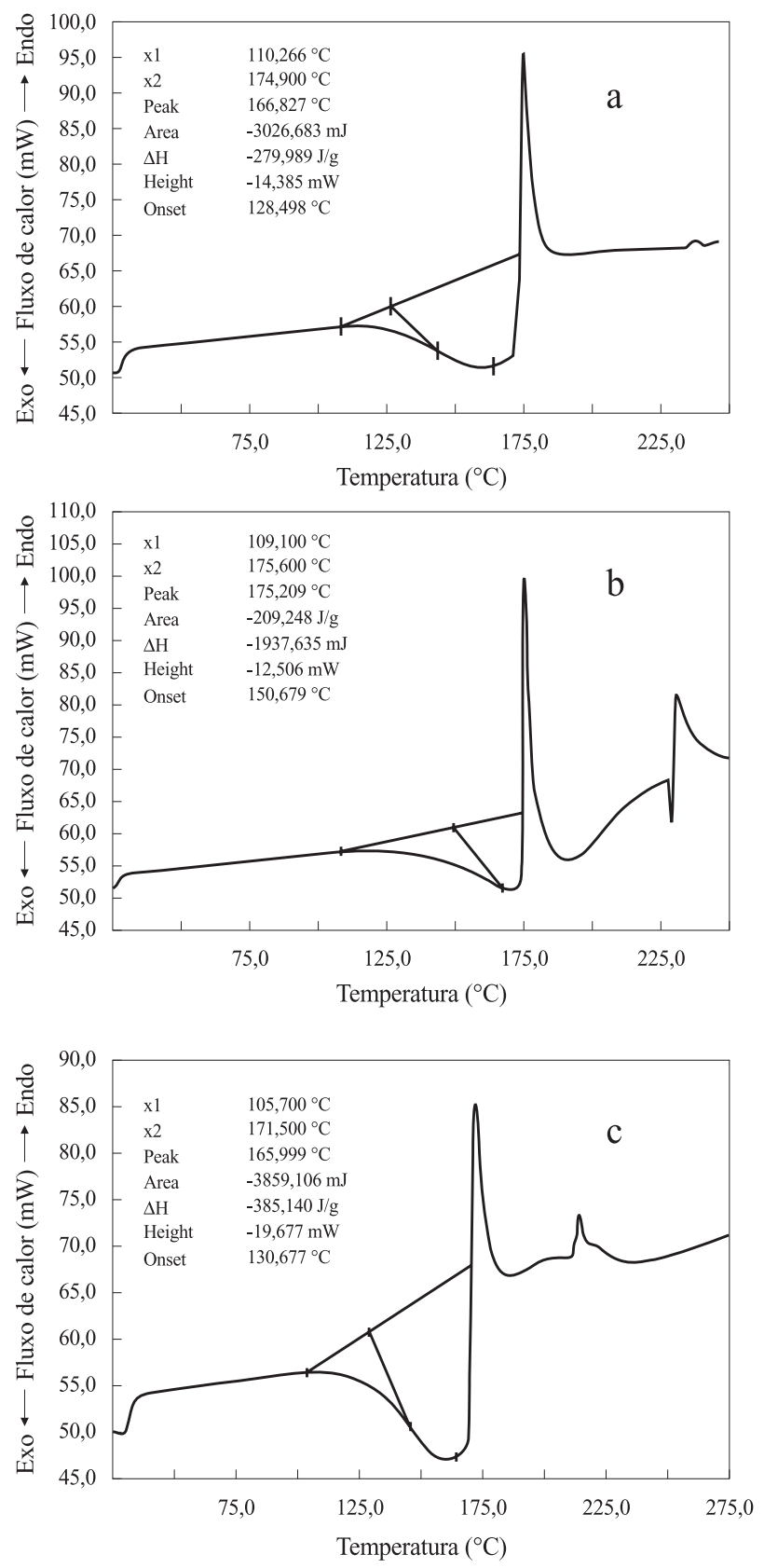

Figura 3. Curvas de DSC para as resinas resol A (a), novolaca B (b) e novolaca $\mathrm{C}$ (c).

ção das isotermas reológicas foram de 100 e $110^{\circ} \mathrm{C}$.

A Figura 4 apresenta os reogramas isotérmicos a $100^{\circ} \mathrm{C}$, para as resinas fenólicas. As três amostras estudadas apresentaram valores próximos de tempos de gelificação ( 7400 s). Esta região de gelificação foi obtida pelo cruzamento de G' (módulo de armazenamento) com G" (módulo de perda). Antes de ser atingida a região de gelificação, todos os reogramas apresentaram comportamento Newtoniano, evidenciado pela linearidade dos valores dos módulos de armazenamento e de perda. Este comportamento é característico de resinas que apresen- 

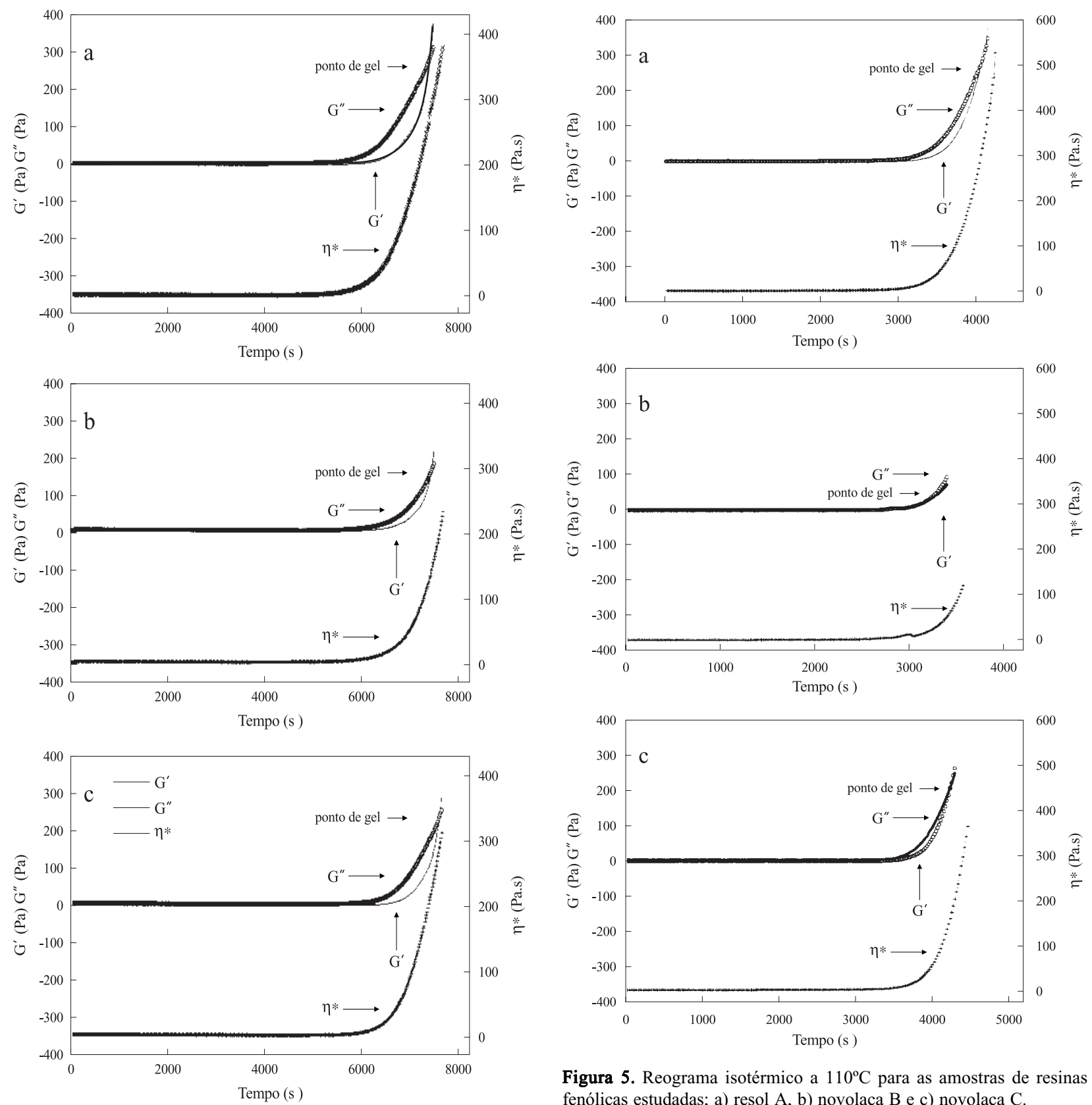

Figura 5. Reograma isotérmico a $110^{\circ} \mathrm{C}$ para as amostras de resinas fenólicas estudadas; a) resol A, b) novolaca B e c) novolaca C.

Figura 4. Reograma isotérmico a $100^{\circ} \mathrm{C}$ para as amostras de resinas fenólicas estudadas, a) resol A, b) novolaca B e c) novolaca C.

tam baixo valor de peso molecular (menor que 3.000 $\mathrm{g} / \mathrm{mol}$ )[7], confirmado pelas análises de SEC das amostras em estudo.

Entretanto, as análises reológicas feitas a $110^{\circ} \mathrm{C}$ das resinas A, B e C (Figura 5) mostraram tempos de gelificação menores e com diferenças mais significativas, quais sejam 4060, 3199 e 4264 s, respectivamente. Este fato se deve à cinética de cura mais rápida a $110^{\circ} \mathrm{C}$ do que à $100^{\circ} \mathrm{C}$.

Para todas as amostras em estudo, no início da análise observou-se um valor nulo do módulo elásti-

co de relaxação (região em que o sistema possui comportamento Newtoniano). Devido à escala dos gráficos apresentados, tanto o módulo de armazenamento quanto o módulo de perda apresentaram um comportamento linear até atingirem a região de gelificação. No entanto, quando a escala é expandida, observa-se um aumento contínuo dos valores de G" com o tempo de análise.

Próximo à região de gel verificou-se um rápido crescimento do módulo de armazenamento quando comparado ao módulo de perda, caracterizando uma mudança para um sistema viscoelástico. Este fato se deve ao aumento da componente real da viscosidade 
Tabela 3. Tempos de início de viscosidade complexa para as amostras das resinas fenólicas estudadas.

\begin{tabular}{ccccc}
\hline Amostra & $\begin{array}{c}\text { Tempo de evolução } \\
\left(\mathbf{1 0 0}^{\mathbf{0}} \mathbf{C}\right)(\mathbf{s})\end{array}$ & $\begin{array}{c}\text { Dif. de tempo* } \\
(\mathbf{\%})\end{array}$ & $\begin{array}{c}\text { Tempo de evolução } \\
\left(\mathbf{1 1 0}^{\mathbf{O}} \mathbf{C}\right)(\mathbf{s})\end{array}$ & $\begin{array}{c}\text { Dif. de tempo* } \\
(\mathbf{\%})\end{array}$ \\
\hline Resol A & 6000 & - & 3400 & - \\
Novolaca B & 6200 & 3,34 & 3000 & 11,8 \\
Novolaca C & 6100 & 1,67 & 2700 & 20,6 \\
\hline
\end{tabular}

* Diferença de tempo de início de evolução da curva de viscosidade complexa (Resol = referência)

$h^{\prime}\left(h^{\prime}=G\right.$ '”/w, onde w é a velocidade angular do prato superior), devido ao aumento do peso molecular.

A discussão apresentada é confirmada pela evolução da viscosidade complexa, caracterizada pelo aumento da densidade de ligações cruzadas do polímero. O menor aumento da viscosidade complexa no início da análise é característico de uma estruturação mais lenta do sistema. Apesar da evolução da viscosidade complexa fornecer dados referentes à região de gel, o presente trabalho adotou o cruzamento de G' e G", pelo fato dos dados obtidos serem mais precisos.

A Tabela 3 apresenta os valores de início da evolução da viscosidade complexa $\eta$ para as amostras de resinas fenólicas analisadas, a 100 e $110^{\circ} \mathrm{C}$. Observouse que, as amostras de resinas fenólicas tratadas a $110^{\circ} \mathrm{C}$, após o tempo de análise de 3000 segundos, teve a evolução do módulo de perda (componente viscosa) antes da evolução do módulo de armazenamento (componente elástica).

Nas análises realizadas a $100^{\circ} \mathrm{C}$ não ocorreu uma variação significativa no tempo de início de evolução das curvas de viscosidade complexa, que se manteve próximo de 6000 segundos para todas as amostras com diferenças entre os tempos de evolução de 1,6 a $3,3 \%$ (Tabela 3). Entretanto, este fato não é observado nas análises realizadas a $110^{\circ} \mathrm{C}$, devido a esta temperatura acelerar a cinética de polimerização das resinas, mostrando diferenças entre os tempos de evolução de 11 a 20\% (Tabela 3).

Levando-se em consideração as etapas envolvidas no processo de impregnação das pré-formas de carbono, como: a eliminação do ar presente no reforço via vácuo, o aquecimento da resina, a impregnação do reforço e a aplicação de pressão, a temperatura de $100^{\circ} \mathrm{C}$ mostrou-se a mais adequada, permitindo uma maior flexibilidade para o ajuste dos parâmetros de processo.

\section{Conclusões}

A resina fenólic esol A apresentou as melhores características para ser utilizada como impregnante no processamento de compósitos carbono/fenólica e, consequentemente, no de compósitos carbono/carbono. Esta escolha se baseou na maior porcentagem de carbono fixo associada a um valor de viscosidade adequado à impregnação do reforço de fibras de carbono.

As análises térmicas via calorimetria exploratória diferencial permitiram observar que a temperatura de início da reação de polimerização é de, aproximadamente, $110^{\circ} \mathrm{C}$. E, as análises reológicas mostraram que a temperatura de $100^{\circ} \mathrm{C}$ é a mais adequada para a impregnação da resina fenólica ao reforço, devido ao maior tempo para atingir a região de gelificação ( $\sim 6000$ s), permitindo uma maior flexibilidade no ajuste dos parâmetros de processo do compósito.

\section{Agradecimentos}

Os autores agradecem ao CNPq e ao Ministério da Aeronáutica pelo apoio financeiro, ao Departamento de Engenharia de Materiais da UFSCar por viabilizar as análises reológicas, ao Instituto de Química de São Carlos/USP pelas análises cromatográficas e às empresas Resana Ind. Químicas e Schenectady do Brasil Ltda. pelo fornecimento das amostras.

\section{Referências Bibliográficas}

1. Savage, G. - "Carbon-carbon composites", Chapman \& Hall, London (1993).

2. Jenkins, G. M. \& Kawamura, K. - "Polymeric carbons: carbon fibre, glass and char", Cambridge Univ. Press (1976).

3. Marsh, H. - "Introduction to carbon science", Butterworths \& Co Ltda (1989).

4. Thomas, C. R. - "Essentials of carbon-carbon composites", Cambridge (1993).

5. Segal, C. L. - SAMPE Journal, 32 (4), p. 12, (1996). 
6. Otani, S. - "Estudo da influência das características dos piches de alcatrão de hulha na obtenção de compósitos $C / C$ ", Tese de Doutorado, Universidade de São Paulo, Brasil (1996).

7. Botelho, E.C. - "Estudo reológico de resinas fenólicas e furfurílica na obtenção de materiais carbonosos", Dissertação de Mestrado, Instituto Tecnológico de Aeronáutica, Brasil (1998).

8. Siegfried, K. J. - Encyclopedia of Polym. Sci. Tech., 7, p. 432, Herman S. Mark, Norman G. Gaylord, Norbert M. Bikales, Jonh Wiley and Sons, N. York (1985).

9. Ferreira, S. R. - "Estudos do processo de obtenção e caracterização das propriedades mecânicas de conjugados C/C tridimencionais", Dissertação de Mestrado, Instituto Tecnológico de Aeronáutica, Brasil (1992).

10. Costa, L., Montelera, L. R., Camino, G., Weil, E. D., Pearce, E. M. - Polymer - 56, p.23 (1997).

11. Taylor, R. E. \& Kline, D. E. - Carbon, 5, p.607 (1967).

12. Mijovic, J. \& Schafran B. - SAMPE Journal, 26 (3), p. 51 (1990).

13. Bhatia, G., Aggarwal, R.K., Chari, S.S., Jain, G.C. - Carbon, 15 (4), p. 219 (1977).

14. Bird, R. B. - "Dynamics of polymeric líquids: Fluid mechanics", 2a.ed., 1, Wiley-Interscience Publication (1987).

15. Nielsen, L E. - "Polymer rheology, Monsanto Company”, Marcel Dekker, St. Louis, Missouri (1977).

16. Yanovsky, Y. G. - "Polymer rheology theory and practice", Russian Academy of Sciences, Moscow, Rússia (1993).

17. Cross, M. M. - Rheological Acta, 18, 5, p. 609 (1979).
18. Haügen, P. \& Tung, M. A. - Journal Inst. Can. Science Technol. Aliment, 9 (2), p 98 (1976).

19. White, J. L., Gopalakrishnan, M. K., Fathollahi, B. A. - Carbon, 32 (2), p. 301 (1994).

20. Grenier-Loustalot, M. F., Larroque, S., Grenier, P., Leca, J. P., Bedel, D. - Polymers, 35 (14), P.3046 (1994).

21. Knop, L. A. P. - "Phenolic resins", SpringerVerlag, Berlin Heidelberg (1985).

22. Mano, E. B. - "Introdução a polímeros", Edgard Blücher (1985).

23. Conley, R. T - Journal of Applied Polymer Science, 7, p. 171 (1963).

24. Hadad, D. K. \& May, C. A. - "Engineered materials handbook", p. 517, ASM International Handbook Committee, Metals Park, $\mathrm{OH}$ (1988).

25. Balduhn, R. \& Fitzer, E. - Carbon, 18 (155), p. 155 (1980).

26. Cahn, R., Haasen, W. P, Kramer, E. J. - Glasses and Amorphous Materials, 9, p. 559 (1991)

27. Berglund L. A. \& Kenny, J. M. - SAMPE Journal, 27 (2), p. 27 (1991).

28. Peer, H. G. - Recuel, 75, p. 851 (1959).

29. Annual American Standard Test Methods. ASTM D 2415-66 (0404), Philadelphia, p. 180 (1988).

30. Annual American Standard Test Methods, ASTM D 3175-73 (0505), Philadelphia, p. 300 (1988).

31. Annual American Standard Test Methods, ASTM D 2416-84 (0404), Philadelphia, p. 182 (1988).

Recebido: 31/07/98

Aprovado: 22/01/99 